\title{
Apparent radii of neutron stars and equation of state of dense matter
}

\author{
P. Haensel ${ }^{\star}$ \\ N. Copernicus Astronomical Center, Polish Academy of Sciences, Bartycka 18, 00-716 Warszawa, Poland \\ Received 20 July 2001 / Accepted 20 September 2001

\begin{abstract}
Apparent (radiation) radius of neutron star, $R_{\infty}$, depends on the star gravitational mass in quite a different way than the standard coordinate radius in the Schwarzschild metric, $R$. We show that, for a broad set of equations of state of dense matter, $R_{\infty}\left(M_{\max }\right)$ for the configurations with maximum allowable masses is very close to the absolute lower bound on $R_{\infty}$ at fixed $M$, resulting from the very definition of $R_{\infty}$. Also, the value of $R_{\infty}$ at given $M$, corresponding to the maximum compactness (minimum $R$ ) of neutron star consistent with general relativity and condition $v_{\text {sound }}<c$, is only $0.6 \%$ higher than this absolute lower bound. Theoretical predictions for $R_{\infty}$ are compared with existing observational estimates of the apparent radii of neutron stars.
\end{abstract}

Key words. dense matter - equation of state - stars: neutron - stars

\section{Introduction}

Measuring the spectrum of radiation from neutron star surface (or, more precisely, atmospheres), combined with knowledge of distance, enables one, assuming spherical symmetry, to determine total luminosity, effective surface temperature, and neutron star radius. Recently, such studies have been carried out for Geminga (Golden \& Shearer 1999) and RX J185635-3754 (Walter 2001); distance from these isolated neutron stars was obtained from measuring of the annual parallax. Very recently, Chandra observations of X-ray sources in the globular clusters (whose distance is known with relatively good precision) were proposed (and applied) to calculate the radius of a neutron star in quiescence (Rutledge et al. 2001).

Neutron star are relativistic objects, and for masses above solar mass their radii may be only 1.5-2 times larger than the gravitational (Schwarzschild) radius $r_{\mathrm{g}} \equiv$ $2 G M / c^{2}=2.95\left(M / M_{\odot}\right) \mathrm{km}$. Therefore, because of a sizable spacetime curvature close to neutron star, one has to distinguish between the "true" or "coordinate" radius, $R$, which is the radial coordinate of the stellar surface in the Schwarzschild metric, and the "apparent radius" (sometimes called "radiation radius"), $R_{\infty}$, as determined by a distant observer studying radiation from neutron star surface.

In the present letter we calculate dependence of $R_{\infty}$ on neutron star mass for a broad set of equations of state of dense matter. We discuss properties of the theoretical $R_{\infty}(M)$ curves, and finally we confront theoretical

\footnotetext{
* e-mail: haensel@camk.edu.pl
}

calculations with recent observational estimates of apparent radii of neutron stars.

\section{2. $R(M), R_{\infty}(M)$, and their theoretical lower bounds}

The effective surface temperature, $T_{\mathrm{s}}$, at the neutron star surface, is related to total photon luminosity, $L_{\gamma}$, by

$L_{\gamma}=4 \pi R^{2} \sigma_{\mathrm{SB}} T_{\mathrm{s}}^{4}$,

where all quantities are measured by a local observer on neutron star surface. Spherical symmetry is assumed. A distant observer ("at infinity") will measure "apparent luminosity" $L_{\gamma}^{\infty}$, "apparent effective temperature" $T_{\mathrm{s}}^{\infty}$, and "apparent radius" $R_{\infty}$, related to quantities appearing in Eq. (1) by (Thorne 1977)

$L_{\gamma}^{\infty}=L_{\gamma}\left(1-\frac{r_{\mathrm{g}}}{R}\right)=4 \pi R_{\infty}^{2} \sigma_{\mathrm{SB}}\left(T_{\mathrm{s}}^{\infty}\right)^{4}$

$T_{\mathrm{s}}^{\infty}=T_{\mathrm{s}} \sqrt{1-\frac{r_{\mathrm{g}}}{R}}, \quad R_{\infty}=\frac{R}{\sqrt{1-r_{\mathrm{g}} / R}}$.

As we will show, dependence of $R_{\infty}$ on neutron star mass differs considerably from $R(M)$; the difference, which reflects spacetime curvature near neutron star, increases with increasing $M$, and becomes quite large at the maximum allowable mass, $M_{\max }$. The curves $R(M)$ and $R_{\infty}(M)$, calculated for a broad set of equations of state (EOS) of dense matter, are presented in Figs. 1 and 2, respectively. One notices, that for moderately stiff and stiff equations of state $\left(M_{\max } \gtrsim 1.8 M_{\odot}\right)$ without a strong 


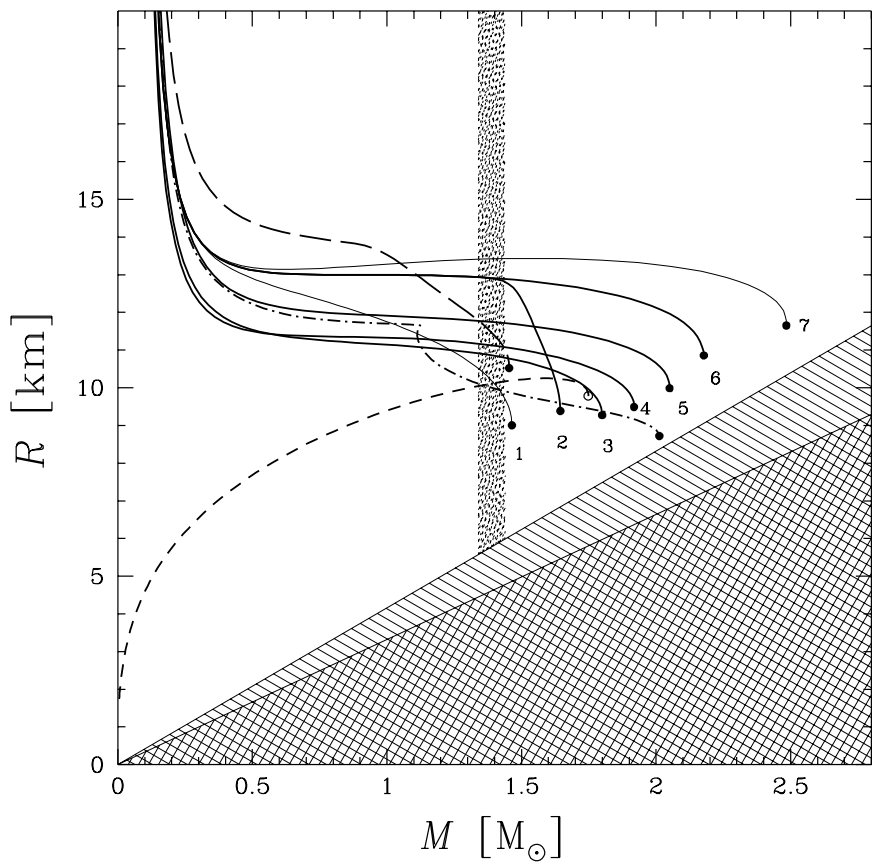

Fig. 1. Neutron star radius $R$ versus gravitational mass $M$, for seven EOS of baryonic matter, labeled by numbers 1-7. 1: BPAL12 of Bombaci et al. (1995); 2: EoSN1H1 of Balberg et al. (1999); 3: FPS of Pandharipande \& Ravenhall (1989); 4: Baldo et al. (1997); 5: Douchin \& Haensel (2000); 6: EoSN1 of Balberg et al. (1999); 7: EoSN2 of Balberg et al. (1999). Dotted line corresponds to strange stars built of self-bound quark matter (SQ1, Haensel et al. 1986). Long dashes: hybrid neutron stars of dense matter with a mixed baryonquark phase, EOS from Table 9.1 of Glendenning (1997). Long dashes-dot line: EOS with first-order phase transition to a pure kaon-condensed matter (Kubis 2001). Doubly hatched area is prohibited by general relativity and corresponds to $R<\frac{9}{8} r_{\mathrm{g}}$. Singly hatched area is excluded by general relativity combined with condition $v_{\text {sound }}<c$. In the case of stars built of baryonic matter, configurations with maximum allowable mass is indicated by a filled circle, and in the case of strange stars, built of self-bound quark matter - by an open circle. Shaded vertical band corresponds to the range of precisely measured masses of binary radio pulsars.

softening at highest densities, for $M \gtrsim 0.5 M_{\odot}$ the apparent radius $R_{\infty}$ increases with increasing $M$ (except for a tiny region close to $M_{\max }$ ), in contrast to $R(M)$, which decreases in the same mass interval.

In Fig. 1, straight lines, marking upper boundaries of the hatched regions of the $R-M$ plane, result from quite general physical conditions imposed on the configurations of hydrostatic equilibrium in general relativity. The lower boundary results from the condition that pressure within an equilibrium configuration should be finite, and can be expressed as $R(M)>\frac{9}{8} r_{\mathrm{g}}$ (Buchdahl 1959; general proof can be found in Weinberg 1972). This condition can rewritten as $R(M)>R_{\min 1}(M)=3.32\left(M / M_{\odot}\right) \mathrm{km}$. A stronger condition is obtained if we additionally require that sound speed within the star should be subluminal: $v_{\text {sound }}=\sqrt{\mathrm{d} P / \mathrm{d} \rho}<c$ (such a condition is

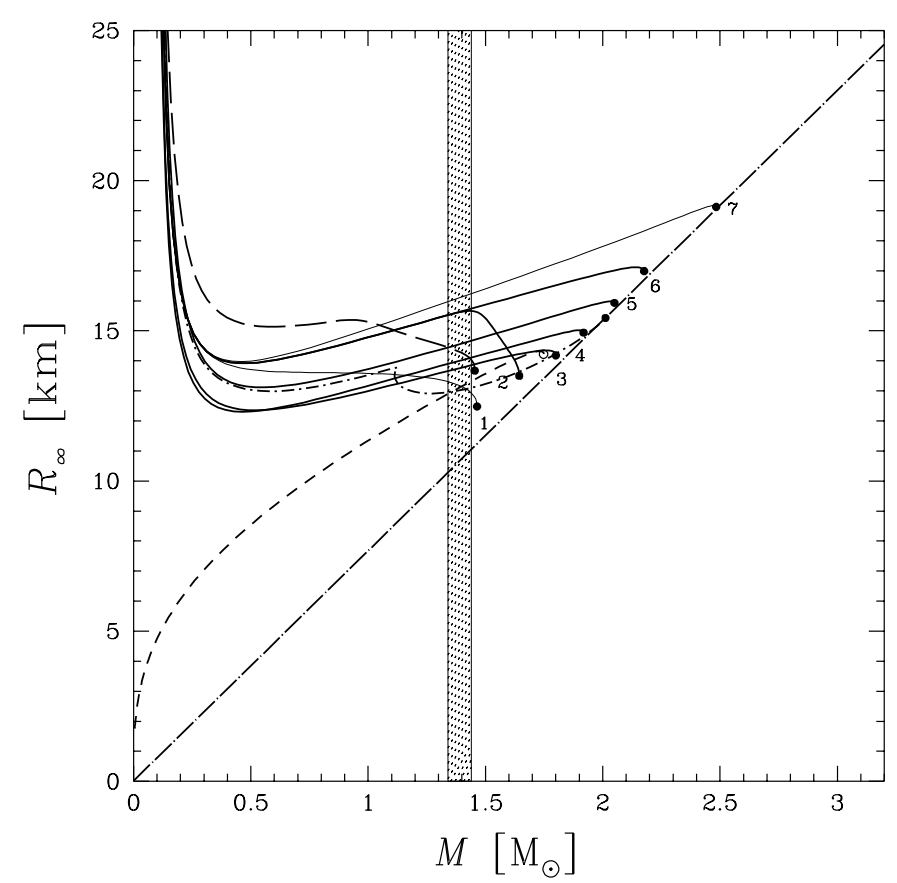

Fig. 2. Apparent radius of neutron star, $R_{\infty}$, versus gravitational mass, $M$. Notation as in Fig. 1. Thick long-dash - dot straight line corresponds to minimum $R_{\infty}$ at a given $M$.

necessary, but not sufficient (Olson 2000), to respect causality in a fluid medium). The condition $v_{\text {sound }}<c$ implies a lower bound on $R$ at a given $M$ (Lindblom 1983; Haensel \& Zdunik 1989; Lattimer et al. 1990; Glendenning 1992; Haensel et al. 1999; Glendenning 2000). In what follows, we will use numerical values of the absolute lower bound $R_{\min 2}(M)=4.17\left(M / M_{\odot}\right) \mathrm{km}$, as calculated in Haensel et al. (1999); the older values obtained in (Lattimer et al. 1990; Glendenning 1992) are slightly higher, because of the assumed presence of an outer envelope (crust) in neutron star models, while the value deduced from (Lindblom 1983) is not very precise (see Haensel et al. 1999).

A strict lower bound on $R_{\infty}(M)$ results from the very definition of $R_{\infty}$ (Lattimer \& Prakash 2001). Namely, the definition of $R_{\infty}$ implies

$\frac{R_{\infty}}{r_{\mathrm{g}}}=\frac{R}{r_{\mathrm{g}} \sqrt{1-r_{\mathrm{g}} / R}}$.

The right-hand-side of the above equation is a function of $x \equiv r_{\mathrm{g}} / R$ only. It diverges to $+\infty$ at $x=0$ and at $x=1$. At fixed $M$, it has a single minimum at $x=2 / 3$. Therefore, minimum value of $R_{\infty}(M)$ is $R_{\infty, \min }(M)=$ $7.66\left(M / M_{\odot}\right) \mathrm{km}$ (Lattimer \& Prakash 2001). Let us notice, that this limiting $R_{\infty}$ for an "apparently most compact" neutron star is very close to (but a little smaller than) that for a maximum compactness $x=0.7081$ consistent with $v_{\text {sound }}<c$, given by $7.71\left(M / M_{\odot}\right) \mathrm{km}$. However, at any $M$ the difference is only $0.6 \%$, and therefore in practice smallest $R$ at a fixed $M$, consistent with $v_{\text {sound }}<c$, can be considered as corresponding to smallest $R_{\infty}(M)$, and vice versa. 
This result can be easily understood, because $x=$ 0.7081 is only by 0.05 higher than $2 / 3$ corresponding to the minimum of $R_{\infty}$. Therefore, relative difference between $R_{\infty, \text { min }}$ and the value corresponding to minimum value of $R$ at given $M$ (assuming $v_{\text {sound }}<c$ ), can be estimated as $\simeq 2.25 \times(0.05)^{2}=0.6 \%$, which is consistent with our exact result.

While the subluminal $\left(v_{\text {sound }}<c\right)$ upper bound on $x$ at given $M$ is slightly larger than $2 / 3$, the actual maximum values of $x$ for various EOS, which are reached at $M_{\max }$ for these EOS, are lower than $2 / 3$. However, if we restrict to medium stiff and stiff EOS, with $M_{\max } \gtrsim 1.8 M_{\odot}$, then $x_{M_{\max }} \simeq 0.6$, which is only 0.06 lower than $2 / 3$. We may therefore expect, that for these EOS, which actually constitute majority of models in Figs. $1,2,\left(R_{\infty}\right)_{M_{\max }}$ will be only $\sim 2.25 \times(0.06)^{2} \simeq 0.8 \%$ larger than $R_{\infty \text {,min }}$. This explains, why for these EOS the points at $M_{\max }$ are so close to the $R_{\infty, \min }(M)$ line in Fig. 2.

As shown by Lattimer \& Prakash (2001), one expects that for any baryonic baryonic EOS, $R_{\infty}>11 \mathrm{~km}$, independently of neutron star mass. Our Fig. 2 confirms this "practical lower bound" on $R_{\infty}$. On the contrary, there is no lower limit on $R_{\infty}$ for bare strange stars, whose size can be as small as hundred fermis. For strange stars covered with a layer of normal matter, minimum radius is reached at $M \sim 0.01 M_{\odot}$, and for a maximally thick crust it is about 5-6 km (see, e.g., Glendenning 1997).

\section{Observational bounds on $\boldsymbol{R}_{\infty}$}

In what follows we will briefly review observational determination of apparent radius of neutron star. We will restrict to cases, which seem to us most promising. In all cases, what one determines is actually $R_{\infty} / d$, where $d$ is neutron star distance. Therefore, independent knowledge of $d$ is mandatory to calculate $R_{\infty}$ from observational data. Generally, after fitting the spectrum of photons emitted from neutron star, one tries to get the interval $R_{\infty, \mathrm{l}}<R_{\infty}<R_{\infty, \mathrm{u}}$, to which the value $R_{\infty}$ belongs at not less than $90 \%$ confidence level. An EOS is considered to be ruled out, if no point on its $R_{\infty}(M)$ curve can satify this condition. In practice, the condition reduces to $R_{\infty}<R_{\infty, \mathrm{u}}$. Generally, conclusions from the application of this criterion should be taken with a grain of salt, because of the difficulty in estimating of the error in the photon spectrum fitting.

\subsection{Close by isolated neutron stars}

At the $95 \%$ confidence level, results of Golden \& Shearer (1999) imply $R_{\infty}<R_{\infty, \mathrm{u}}=17.6 \mathrm{~km}$ assuming the $\mathrm{H}$ atmosphere, and $R_{\infty}<R_{\infty, \mathrm{u}}=16.5 \mathrm{~km}$ for the black body thermal spectrum (which turns out to be practically indistinguishable from the $\mathrm{Fe} / \mathrm{Si}$ model atmosphere spectrum). Therefore, the value of the upper bound on the apparent radius of Geminga is, fortunately, not very sensitive to the assumed atmospheric model.
As for this writing, the case of RX J185635-3754 (Walter 2001, and references therein), is much less clear. Using atmosphere model of the photon spectrum, one deduces from numbers quoted in Walter (2001) the upper bound $R_{\infty, \mathrm{u}}(\mathrm{atm})=18 \mathrm{~km}$ at the $2 \sigma$ confidence level. However, if one uses the black body spectrum model, assuming spherical symmetry, one gets an abnormally small upper bound, $R_{\infty, \mathrm{u}}(\mathrm{BB})=8.4 \mathrm{~km}$ (Walter 2001; notice that we use results at the $2 \sigma$ confidence level). Clearly, these results are very preliminary, and we have still to wait for more reliable and less model dependent determinations of $R_{\infty}$ for this isolated neutron star.

\subsection{X-ray transients in globular clusters}

Very recently, Rutledge et al. (2001) proposed a method of measuring $R_{\infty}$ of neutron stars, observed as X-ray transients in globular clusters. As an example, they studied transcient X-ray source CXOU 132619.7-472910.8 in NGC 5139, fitting photon spectrum with the $\mathrm{H}$ atmosphere model. They obtained, at 90\% confidence level, $R_{\infty}=14.3 \pm 2.5 \mathrm{~km}$ (assuming $10 \%$ uncertainty in the distance to NGC 5139), which results in $R_{\infty, \mathrm{u}}=$ $16.8 \mathrm{~km}$. The advantage of the proposed method stems from the fact, that for neutron stars located in globular clusters both the distance and interstellar hydrogen column density are rather well known.

\subsection{Other estimates of neutron star radii}

They are mostly related to accreting neutron stars, observed as X-ray bursters (see, e.g., Titarchuk 1994; Haberl \& Titarchuk 1995; Burderi \& King 1998; Psaltis \& Chakrabarty 1999; Li et al. 1999a; Li et al. 1999b, and references therein). One has to mention a strong model dependence of theoretical analyses, and frequent neglect (Burderi \& King 1998; Psaltis \& Chakrabarty 1999; Li et al. 1999a; Li et al. 1999b) of the space-time curvature effects, which are actually crucial for the difference between $R$ and $R_{\infty}$.

\subsection{Theory versus observations}

As one can see in Fig. 2, the upper bounds $R_{\infty, \mathrm{u}}$ on the apparent radius of Geminga are consistent with theoretical predictions for $R_{\infty}(M)$, based on considered baryonic EOS of dense matter, provided the neutron star mass is above $0.2 M_{\odot}$.

The value of the upper bound $R_{\infty, \mathrm{u}}=16.8 \mathrm{~km}$, obtained by Rutledge et al. (2001) for the transient X-ray source CXOU 132619.7-472910.8 in NGC 5139, is consistent with neutron star $R_{\infty}(M)$ curves in Fig. 2, provided $M>0.4 M_{\odot}$. In the case of stiffest EOS (with $M_{\max }>2.2 M_{\odot}$ ), the value of $R_{\infty, \mathrm{u}}$ rules out high-mass neutron stars with $M>1.6 \div 2.0 M_{\odot}$.

As for this writing, attempts to estimate the apparent radius of RX J185635-3754 (Walter 2001) are very model 
dependent. Nevertheless, we are tempted to make a following comment. Had we accepted the estimate $R_{\infty}(\mathrm{BB})$ for RX J185635-3754, this object could be but a low-mass strange star. In order to produce thermal photon spectrum, this low-mass strange star should have been covered with a layer of normal matter, because a bare quark surface would be a too weak photon emitter (Chmaj et al. 1991; Usov 2001). One can only hope that more precise measurement of the photon spectrum for this isolated neutron star will liberate us from such basic ambiguities.

\section{Summary}

Detection of photons emitted from the surface of isolated neutron stars of known distance can result in determination of the apparent neutron star radius, $R_{\infty}$. Due to significant space-time curvature, dependence of $R_{\infty}$ on on stellar mass is quite different from that of the standard "coordinate" radius $R$. The very definition of $R_{\infty}$ implies a lower bound, obtained by Lattimer \& Prakash (2001), of $7.66\left(M / M_{\odot}\right) \mathrm{km}$. At any $M$, this lower limit is very close to the value of $R_{\infty}$ corresponding to the minimum $R$, calculated under the condition of subluminal sound. Simultaneously, the actual values of $R_{\infty}$ calculated at the maximum allowable mass are also close to this limit. For moderately stiff and stiff EOS with $M_{\max } \gtrsim 1.8 M_{\odot}$, the actual value of $R_{\infty}\left(M_{\max }\right)$ is less than one percent higher than the absolute lower bound on $R_{\infty}$ at this value of stellar mass.

Most reliable observational estimates of $R_{\infty}$, obtained for Geminga and transient X-ray source CXOU 132619.7472910.8 in NGC 5139, lead to upper bounds on $R_{\infty}$, which are consistent with all considered baryonic EOS, provided the mass of neutron star is above $0.2 \div 0.4 M_{\odot}$.

Acknowledgements. I am grateful to J. L. Zdunik for the reading of the manuscript and for helpful remarks. I am also grateful to A. Potekhin for his precious help in the preparation of figures. This research was partially supported by the KBN grant No. 5P03D.020.20.

\section{References}

Balberg, S., Lichtenstadt, I., \& Cook, G. B. 1999, ApJS, 121, 515

Baldo, M., Bombaci, I., \& Burgio, G. F. 1997, A\&A, 328, 279

Bombaci, I. 1995, in Perspectives on Theoretical Nuclear Physics, ed. I. Bombaci, A. Bonaccorso, A. Fabrocini, et al., 223

Bondi, H. 1964, Proc. Roy. Soc. A, 281, 39
Buchdahl, H. A. 1959, Phys. Rev., 116, 1027

Burderi, L., \& King, A. R. 1998, ApJ, 505, L135

Caraveo, P. A., Bignami, G. F., Mignani, R., \& Taff, L. G. 1996, ApJ, 461, L91

Chmaj, T., Haensel, P., \& Słomiński, 1991, Nucl. Phys. B, 24B, 40

Douchin, F., P., \& Haensel, P. 2000, Phys. Lett. B, 485, 107

Glendenning, N. K. 1992, Phys. Rev. D, 46, 4161

Glendenning, N. K. 2000, Phys. Rev. Lett., 85, 1150

Glendenning, N. K. 1997, Compact Stars: Nuclear Physics, Particle Physics and General Relativity (Springer, New York)

Golden, A., \& Shearer, A. 1999, A\&A, 342, L5

Haberl, F., \& Titarchuk, L. 1995, A\&A, 299, 414

Haensel, P., Zdunik, J. L., \& Schaeffer, R. 1986, A\&A, 160, 121

Haensel, P., \& Zdunik, J. L. 1989, Nature, 340, 617

Haensel, P., Lasota, J. P., \& Zdunik, J. L. 1999, A\&A, 344, 151

Kubis, S. 2001, Ph.D. Thesis, Jagellonian University (unpublished)

Lampton, M., Lieu, R., Schmitt, J. H. M. M., et al. 1997, ApJS, 108,545

Lattimer, J. M., \& Prakash, M. 2001, ApJ, 550, 426

Li, X.-D., Bombaci, I., Dey, M., Dey, J., \& van den Heuvel, E. P. J. 1999a, Phys. Rev. Lett., 83, 3776

Li, X.-D., Ray, S., Dey, J., Dey, M., \& Bombaci, I. 1999b, ApJ, $527, \mathrm{~L} 51$

Lindblom, L. 1984, ApJ, 278, 364

Olson, T. S. 2001, Phys. Rev. C, 63, 015802

Pandharipande, V. R., \& Ravenhall, D. G. 1989, in Proc. NATO Advanced Research Workshop on nuclear matter and heavy ion collisions, Les Houches, 1989, ed. M. Soyeur et al. (Plenum, New York, 1989), 103

Psaltis, D., \& Chakrabarty, D. 1999, ApJ, 521, 332

Rutledge, R. E., Bildsten, L., Brown, E. F., Pavlov, G. G., \& Zavlin, V. E., ApJ, submitted [astro-ph/0105405]

Thorne, K. S. 1977, ApJ, 212, 825

Titarchuk, L. 1994, ApJ, 429, 340

Usov, V. V. 2001, ApJ, 550, L179

Walter, F. M., Wolk, S. J., \& Neuhäuser, R. 1996, Nature, 379, 233

Walter, F. M., \& Matthews, L. D. 1997, Nature, 389, 358

Walter, F. M., \& An, P. 1998, BAAS, 192, 50.04

Walter, F. M., An, P., Lattimer, J., \& Prakash, M. 2000, in Highly Energetic Physical Processes and Mechanisms for Emission from Astrophysical Plasmas, ed. P. C. H. Martens, S. Tsuruta, \& M. A. Weber (ASP Conf. Ser., San Francisco, ASP), IAU Symp., 195, 437

Walter, F. M. 2001, ApJ, 549, 433

Weinberg, S. 1972, Gravitation and Cosmology: Principles and Applications of the General Theory of Relativity (John Wiley \& Sons, New York), Sect. 11.6 